\title{
The quality of YouTube videos on endoscopic third ventriculostomy and endoscopic third ventriculostomy with choroid plexus cauterization procedures available to families of patients with pediatric hydrocephalus
}

\author{
Nicholas Sader, MD, ${ }^{1}$ Abhaya V. Kulkarni, MD, PhD, ${ }^{2}$ Matthew E. Eagles, MD, ${ }^{1}$ Salim Ahmed, MSc, ${ }^{1}$ \\ Jenna E. Koschnitzky, PhD, ${ }^{3}$ and Jay Riva-Cambrin, MD, MSc ${ }^{1}$ \\ 1Department of Clinical Neurosciences, Division of Neurosurgery, Alberta Children's Hospital, University of Calgary, Alberta, \\ Canada; ${ }^{2}$ Division of Neurosurgery, Hospital for Sick Children, University of Toronto, Ontario, Canada; and ${ }^{3}$ Hydrocephalus \\ Association, Bethesda, Maryland
}

\begin{abstract}
OBJECTIVE YouTube has become an important information source for pediatric neurosurgical patients and their families. The goal of this study was to determine whether the informative quality of videos of endoscopic third ventriculostomy (ETV) and endoscopic third ventriculostomy with choroid plexus cauterization (ETV + CPC) is associated with metrics of popularity.

METHODS This cross-sectional study used comprehensive search terms to identify videos pertaining to ETV and ETV + CPC presented on the first 3 pages of search results on YouTube. Two pediatric neurosurgeons, 1 neurosurgery resident, and 2 patient families independently reviewed the selected videos. Videos were assessed for overall informational quality by using a validated 5-point Global Quality Score (GQS) and compared to online metrics of popularity and engagement such as views, likes, likes/views ratio, comments/views ratio, and likes/dislikes ratio. Weighted kappa scores were used to measure agreement between video reviewers.
\end{abstract}

RESULTS A total of 58 videos ( 47 on ETV, 7 on ETV + CPC, 4 on both) of 120 videos assessed met the inclusion criteria. Video styles included "technical" (62\%), "lecture" (24\%), "patient testimonial" (4\%), and "other" (10\%). In terms of GQS, substantial agreement was seen between surgeons (kappa $0.67[95 \% \mathrm{Cl} 0.55,0.80]$ ) and excellent agreement was found between each surgeon and the neurosurgical resident $(0.77[95 \% \mathrm{Cl} 0.66,0.88]$ and $0.89[95 \% \mathrm{Cl} 0.82$, $0.97])$. Only fair to moderate agreement was seen between professionals and patient families, with weighted kappa scores ranging from 0.07 to 0.56 . Academic lectures were more likely to be rated good or excellent $(64 \% \mathrm{vs} 0 \%, p<$ $0.001)$ versus surgical procedure and testimonial video types. There were significant associations between a better GQS and more likes $(p=0.01)$, views $(p=0.02)$, and the likes/dislikes ratio $(p=0.016)$. The likes/views ratio $(p=0.31)$ and comments/views ratio $(p=0.35)$ were not associated with GQS. The number of likes $(p=0.02)$, views $(p=0.03)$, and the likes/dislikes ratio $(p=0.015)$ were significantly associated with video style (highest for lecture-style videos).

CONCLUSIONS Medical professionals tended to agree when assessing the overall quality of YouTube videos, but this agreement was not as strongly seen when compared to parental ratings. The online metrics of likes, views, and likes/dislikes ratio appear to predict quality. Neurosurgeons seeking to increase their online footprint via YouTube would be well advised to focus more on the academic lecture style because these were universally better rated.

https://thejns.org/doi/abs/10.3171/2019.12.PEDS19523

KEYWORDS ETV + CPC; endoscopic third ventriculostomy with choroid plexus cauterization; YouTube; social media; hydrocephalus; pediatric

$\mathrm{P}$ EDIATRIC hydrocephalus is a disorder that affects children worldwide and has an estimated prevalence of 0.5 cases per 1000 live and stillbirths in the United States and Europe ${ }^{6,9,12,16,22}$ Worldwide, the prevalence is estimated to be 88 per $100,000 .{ }^{15}$ In many instances these patients require treatment, and caregivers are faced with deciding on what therapeutic path to pursue. CSF shunts have a long history in the management of pediatric hydrocephalus; however, endoscopic third ventriculostomy (ETV) is an alternative treatment option, which involves

ABBREVIATIONS CPC = choroid plexus cauterization; ETV = endoscopic third ventriculostomy; GQS = Global Quality Score; IQR = interquartile range.

SUBMITTED September 9, 2019. ACCEPTED December 30, 2019.

INCLUDE WHEN CITING Published online March 6, 2020; DOI: 10.3171/2019.12.PEDS19523. 
creating a hole in the floor of the third ventricle, thereby connecting it to the subarachnoid space. However, this surgery is predominantly used in obstructive hydrocephalus, and is most successful in children older than 2 years of age. ${ }^{17,18}$ ETV with choroid plexus cauterization (CPC) was introduced to the United States and Canada in the late 2000s due to failure of ETV in young infants, and has shown moderate success in this population. ${ }^{19,20,30}$ In properly selected patients it is an attractive treatment option because it avoids the complications associated with indwelling CSF shunts.

The internet and social media are open-ended resources for caregivers and patients to access health information. According to the Pew Internet Project, 59\% of all adults in the United States say they used the internet for health information in the past year. ${ }^{10}$ Sixty-nine percent of American adults use at least one social media website. Of these, YouTube (www.youtube.com) is the second largest site behind Facebook. ${ }^{1,23}$ In a study that looked at the preferences among caregivers of children with hydrocephalus, $75.3 \%$ reported searching the internet for hydrocephalus-related information and 59.5\% used social media for hydrocephalus-related purposes, with Facebook and YouTube being used most frequently. Furthermore, $90 \%$ of parents said they would visit neurosurgeon-recommended websites. ${ }^{21}$

YouTube was founded in 2005 and is the internet's most dominant video-sharing website. It allows users around the world to upload and view videos, as well as interact through comments and ratings. YouTube has more than 1.9 billion users and each day people watch more than 1 billion hours of videos, ${ }^{31}$ making it an ideal knowledge translation platform.

ETV and ETV + CPC are done with an endoscope and projected onto a monitor. This is ideally suited for YouTube, because it is possible to display what the neurosurgeon is viewing during the operation. However, as is the case with many websites, there is little in the way of quality control for videos uploaded to YouTube. While there are a number of videos related to ETV + CPC on the video-sharing platform, the subjective quality of these videos has never been evaluated from a professional or parental viewpoint.

The objectives of this study were to examine the video resources available through YouTube to families of patients with pediatric hydrocephalus and to determine whether the informative quality of these videos is associated with viewership or popularity.

\section{Methods}

\section{Search Strategy}

We performed a YouTube video search on June 22, 2017, in which we used the search terms "Endoscopic Third Ventriculostomy + Choroid Plexus Cauterization" and "ETV + CPC." The only search filter used was "sort by" relevance, which is the default filter when doing a YouTube search. It has been noted that $90 \%$ of individuals click on a result within the first 3 pages when searching for something on the internet. ${ }^{14}$ Therefore, only the first 3 pages of videos for each search term were screened, because it was deemed unlikely that caregivers and patients would go beyond those. URLs of the designated videos were recorded for the reviewers. Videos that were non-English, animal studies, and those deemed nonrelevant after initial screening were not included in the study. Duplicated videos were examined once.

\section{Video Quality Scoring and Evaluation}

Our primary objective was to evaluate the informative quality of the videos by using the 5-point Global Quality Score (GQS) developed by Bernard et al. and published in 2007. ${ }^{4} \mathrm{~A}$ score of 5 means that the video had excellent quality and flow and is very useful for patients; a score of 4 means good quality and generally good flow-most of the relevant information is listed, but some topics are not covered-useful for patients; 3 means moderate quality and suboptimal flow-some important information is adequately discussed but other topics are poorly discussed-somewhat useful for patients; 2 means generally poor quality and poor flow-some information listed but many important topics are missing-of very limited use to patients; and 1 means poor quality and poor flow of the video-most information is missing - not at all useful for patients. This measure has previously been used to evaluate the quality of YouTube videos in a medical setting. ${ }^{5}$ In addition to the GQS, we modified a scoring system published by Sorensen et al. in 2014 to evaluate how useful each video would be for patients and their families. ${ }^{27} \mathrm{Cat}-$ egories within this scoring system included visual and audio production quality, accuracy/usefulness, and comprehensiveness. Last, we used an adaptation of the HONcode principles to further assess video quality. ${ }^{28}$ The HONcode is the oldest, most used, ethical, and trustworthy code for medical and health-related information on the internet. ${ }^{13}$

The videos were reviewed independently and in triplicate by 3 professionals. Two were pediatric neurosurgeons (J.R.C. and A.V.K.) from separate institutions, and 1 was a neurosurgery resident (N.S.), using the 3 scoring systems. Furthermore, 2 geographically separate patient families evaluated each video independently using the GQS. They had been contacted by the National Director of Research Programs (J.E.K.) at the Hydrocephalus Association, a patient-advocacy group, about participating in the study.

\section{Online Video Popularity and Viewer Engagement Metrics}

For each video that met the inclusion criteria, the primary metric was the total number of views and was recorded as of June 22, 2017. Secondary online metrics aimed to assess the level of video engagement by the viewer and included the number of likes, dislikes, comments, as well as more sophisticated ratios used by the commercial department of YouTube such as the like/dislike ratio, the likes/views ratio, and the comments/views ratio. The likes/dislikes ratio identifies whether a video has a more positive or negative impression on viewers. According to online content, having at least 10 likes for every 1 dislike is considered a successful benchmark. ${ }^{25}$ In addition, the likes/views ratio assesses not only if individuals click on the video, but also take the time to inform whether they like the content. According to online content, having at least 4 likes for every 100 views is an acceptable goal. ${ }^{24}$ 
The comments/views ratio identifies whether individuals are taking the time to watch and interact with others by leaving comments. According to online content, having 5 comments for every 100 views is favorable. ${ }^{24}$ Video duration and date of video upload were also recorded.

\section{Video Style Categories}

The videos that met the inclusion criteria were placed into 4 categories including "Lecture Type," "Technical," "Patient Testimonial," and "Other", as determined by 2 of the authors (N.S. and J.R.C.). "Lecture Type" included videos that included an expert leading a discussion of the surgical procedure as well as its indications, potential complications, and/or variations. In the "Technical" category were videos that showed the actual surgical procedure and were either narrated (less common) or not. Videos that were uploaded by patients and/or their families discussing their impressions of the procedure were placed in the "Patient Testimonial" category. Finally, in the "Other" category were videos that did not fit in the previous 3 categories. For example, one video demonstrated an ETV on a brain simulator.

\section{Statistical Analyses}

Continuous variables were reported as medians with interquartile ranges (IQRs). The only exceptions were the popularity metrics in Table 1, which were presented in means and ranges to give the reader a better sense of the range of responses to these neurosurgical videos. Categorical variables were reported as frequencies and percentages. Weighted kappa estimates and their 95\% CIs were reported as the principal measure of interrater agreement because the primary outcome of GQS was an ordinal categorical variable. Four relationships between raters were examined and included: neurosurgeon A versus neurosurgeon B (interprofessional); neurosurgeon A versus a neurosurgery resident (professional-trainee); neurosurgeon A versus parent A (professional-consumer); and parent A versus parent B (interconsumer). Agreement was considered substantial to almost perfect (kappa 0.61-1.00), moderate (kappa 0.41-0.60), and none to fair (kappa 0.000.40 ). Other specific video quality measures (visual and audio production quality, comprehensiveness, and accuracy) were also assessed using weighted kappa and 95\% CIs.

The metrics of popularity such as likes, dislikes, views, number of comments, and the secondary ratios thereof were not normally distributed, with a large right tail of outliers. Therefore, the comparisons of these metrics associations with both 1) overall video quality (GQS) as well as 2) the video style variables were conducted nonparametrically using the Kruskal-Wallis test (1-way ANOVA on ranks). The $\mathrm{p}$ values were reported with $<0.05$ being the cutoff for statistical significance.

Global Quality Scores were then dichotomized into good to excellent quality (GQS 4 and 5) versus low quality (GQS 1,2, and 3). The association between video style and dichotomized GQS (video quality) was analyzed via the Kruskal-Wallis test. All analyses were conducted by SAS (version 9.4, SAS Institute). This cross-sectional study
TABLE 1. Characteristics and metrics of popularity in 58 videos as of June 22, 2017

\begin{tabular}{cc}
\hline \multicolumn{1}{c}{ Variables } & Values \\
\hline Surgical procedure (no., \%) & $47(81 \%)$ \\
\hline ETV & $7(12.1 \%)$ \\
\hline ETV + CPC & $4(6.9 \%)$ \\
\hline Both & $36(62.1 \%)$ \\
\hline Video styles (no., \%) & $14(24.1 \%)$ \\
\hline Technical & $2(3.5 \%)$ \\
\hline Lecture & $6(10.3 \%)$ \\
\hline Testimonial & $7.9(0.4-53.0)$ \\
\hline Other & \\
\hline Mean video length in mins (range) & $31(53.5 \%)$ \\
\hline HON code (no., \%) & $20(34.5 \%)$ \\
\hline Authoritative & $56(96.6 \%)$ \\
\hline Complementary & $10(17.2 \%)$ \\
\hline Privacy & $7(12.1 \%)$ \\
\hline Attribution & $7(12.1 \%)$ \\
\hline Justifiability & $1(1.7 \%)$ \\
\hline Transparency & $3(5.2 \%)$ \\
\hline Advertising policy & $16.2(0-548)$ \\
\hline Financial disclosures & $0.57(0-15)$ \\
\hline Likes (mean, range) & $1.8(0-67)$ \\
\hline Dislikes (mean, range) & $4424(8-114,884)$ \\
\hline Comments (mean, range) & \\
\hline Views (mean, range) & \\
\hline$H O N=$ &
\end{tabular}

HON $=$ Health on the Net.

used publicly accessible data and was exempt from our Research Ethics Board's approval.

\section{Results}

The results of the search strategy are summarized in Fig. 1. Overall, a total of 120 videos were identified. Of these, 2 videos were non-English, 49 nonrelevant, and 1 was a duplicate. This left 68 videos between both search terms. Ten videos were present in both searches, leaving a total of 58 videos for review. Forty-seven videos related to ETV, 7 to ETV + CPC, and 4 to both.

\section{Video Characteristics and Metrics}

The video styles and metrics of popularity are outlined in Table 1. Technical-style videos were the most common (62\%). Lecture-based videos were the next most frequent (24\%), followed by other (10\%) and patient testimonial $(4 \%)$. The mean number of views was 4424 , with the mostwatched video having 114,884 views.

Applying the HONcode principles demonstrated that only $5 \%$ of videos clearly identified funding sources and only $2 \%$ of videos outlined if an advertising company supported the video. ${ }^{19}$ Twelve percent of videos offered justifiability of the claims that the benefits, behavior, interventions, treatments, or products were supported by evidence through HTML links or cited literature. A majority of the 


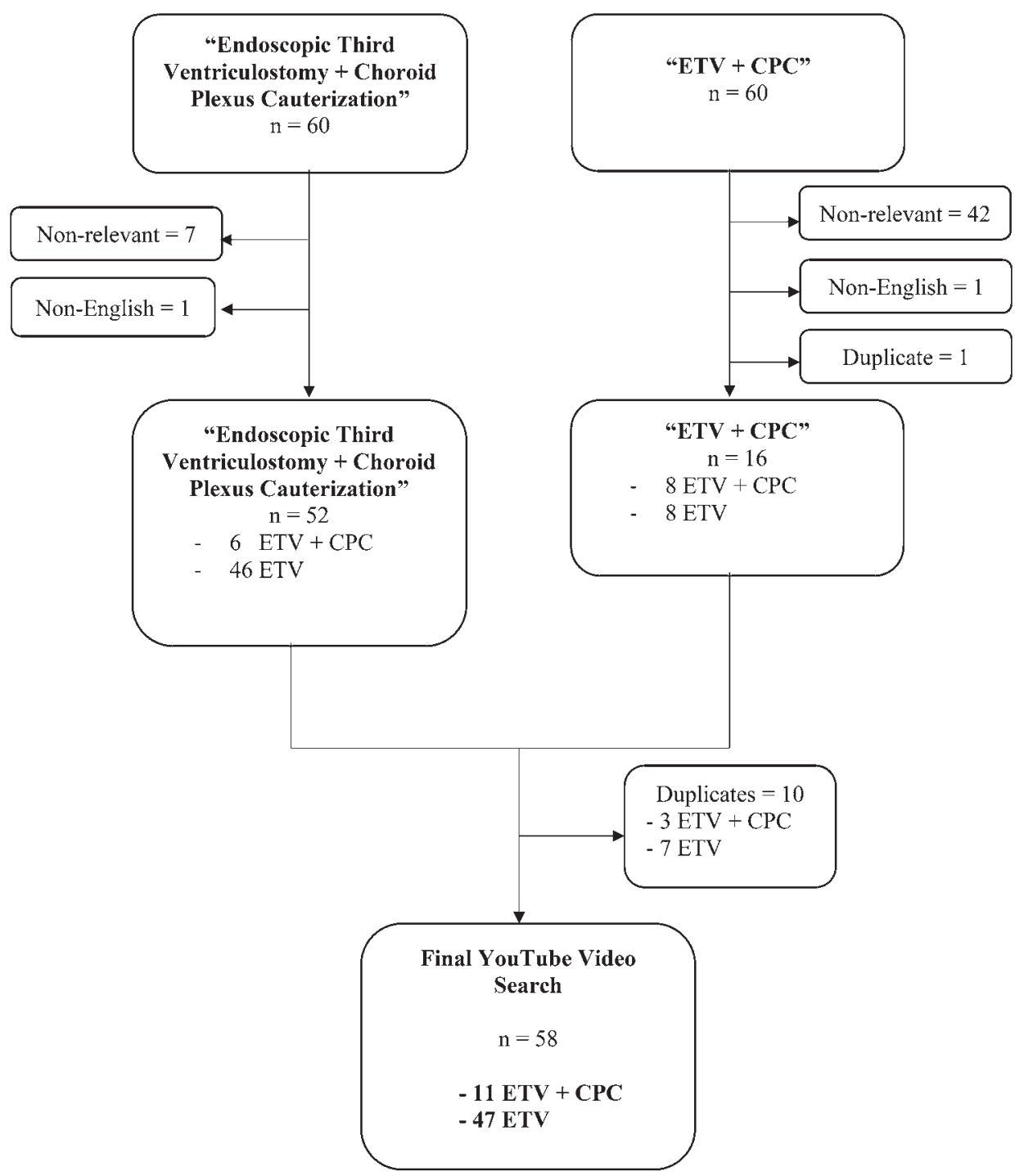

FIG. 1. Flow diagram of YouTube video search results.

videos (54\%) came from a qualified health professional and $97 \%$ of videos maintained the right to confidentiality of and respect for the individual patient featured.

The majority of the videos (60\%) inferred their intended goals, whereas $26 \%$ stated them clearly and explicitly, and video goals were vague and unclear in $16 \%$. Ninetyseven percent of videos were rated as fair and balanced, whereas the indications for and risks of the surgical procedure were only addressed in $26 \%$ and $14 \%$ of videos, respectively.

\section{Interrater Agreement on Video Quality}

Table 2 outlines the weighted kappa values with 95\% CIs for 4 of the relationships between the 5 reviewers by using the GQS and 4 other video quality measures (visual and audio production quality, comprehensiveness, and accuracy). There was substantial agreement on video comprehensiveness, accuracy, GQS, and audio production quality between neurosurgeons. However, the neu- rosurgeons only moderately agreed on visual production quality. Moreover, there was substantial to near perfect agreement on all video quality aspects between the neurosurgery resident and neurosurgeon A. Similar results were seen between neurosurgeon B and the resident trainee (results not shown). In terms of the parents of children with hydrocephalus, they had only slight to fair agreement with the neurosurgeons on all other aspects of video quality but were in moderate agreement in terms of overall quality (GQS) and audio quality. In terms of visual quality, there was slight agreement between the neurosurgeon and parent. Neurosurgeons are aware that the endoscope video output can appear "grainy," which might not be known by parents, and might explain this incongruity. Parents had only fair to moderate agreement with each other, again with the exception of audio production quality. Figure 2 demonstrates the comparison of professionals and parents with regard to the mean frequency of GQS values, with parents tending to give a more extreme GQS. 
TABLE 2. Agreement between stakeholders on different measures of video quality

\begin{tabular}{lcccc}
\hline \multicolumn{1}{c}{ Rating Categories } & $\begin{array}{c}\text { Neurosurgeon A vs } \\
\text { Neurosurgeon B }\end{array}$ & $\begin{array}{c}\text { Neurosurgeon A vs } \\
\text { Resident }\end{array}$ & $\begin{array}{c}\text { Neurosurgeon A vs } \\
\text { Parent A }\end{array}$ & Parent A vs Parent B \\
\hline $\begin{array}{l}\text { Production quality, visual; 3 categories_good, fair, poor } \\
\text { Production quality, audio; 3 categories_good, fair, poor }\end{array}$ & $0.49(0.34,0.63) \dagger$ & $0.85(0.75,0.96)^{*}$ & $0.14(0.02,0.27) \ddagger$ & $0.44(0.25,0.64) \dagger$ \\
\hline $\begin{array}{l}\text { Comprehensiveness; } 4 \text { categories_complete, mostly, } \\
\text { fairly, incomplete }\end{array}$ & $0.65(0.52,0.79)^{*}$ & $0.91(0.83,0.99)^{*}$ & $0.38(0.15,0.62) \ddagger$ & $0.24(0.07,0.42) \ddagger$ \\
\hline $\begin{array}{l}\text { Accuracy/usefulness; } 4 \text { categories_excellent, good, } \\
\text { limited, no use }\end{array}$ & $0.78(0.66,0.89)^{*}$ & $0.90(0.83,0.97)^{*}$ & $0.07(0.01,0.13) \ddagger$ & $0.29(0.13,0.44) \ddagger$ \\
\hline $\begin{array}{l}\text { GQS; } 5 \text { categories-excellent, good, moderate, generally } \\
\text { poor, poor }\end{array}$ & $0.67(0.55,0.80)^{*}$ & $0.89(0.82,0.97)^{*}$ & $0.44(0.25,0.62) \dagger$ & $0.27(0.11,0.43) \ddagger$ \\
\hline
\end{tabular}

Values are expressed as the weighted kappa $(95 \% \mathrm{Cl})$.

${ }^{*}$ Agreement considered substantial, $0.61-0.80$; almost perfect, $0.81-0.99$; or perfect, 1.

$\dagger$ Agreement considered moderate, 0.41-0.60.

$\ddagger$ Agreement considered none, 0 ; slight, $0.01-0.20$; or fair, $0.21-0.40$.

\section{Relationship Between Video Quality (GQS) and Metrics of Popularity and Engagement}

The median likes/dislikes for our sample of ETV and $\mathrm{ETV}+\mathrm{CPV}$ videos was 2 (IQR 0,6), which was below the online threshold for "popularity" of $10 .{ }^{25}$ The median likes/views for our sample was 0.003 (IQR 0, 0.01), which was below the online threshold for "taking the time to inform whether they liked the content" of 0.04. The median comments/views for our sample was 0 (IQR 0,0$)$, which was below the online threshold for "individuals taking the time to watch and interact" of $0.05 .^{24}$

The number of likes $(\mathrm{p}=0.01)$, views $(\mathrm{p}=0.02)$, and likes/dislikes ratio $(p=0.016)$ were found to be significantly associated with overall video quality (GQS). Dislikes $(\mathrm{p}=0.19)$, comments $(\mathrm{p}=0.29)$, the likes/views ratio $(p=0.31)$, and the comments/views ratio $(p=0.35)$ were not associated with GQS.

\section{Video Style and Metrics of Popularity and Engagement}

The number of likes $(p=0.02)$, views $(p=0.03)$, and likes/dislikes ratio (0.015) were significantly associated with video style (Table 3). Specifically, lecture-style videos $(n=14)$ had significantly higher likes, views, and likes/dislikes ratios and included the most "liked" video in the cohort, with 548 likes. One lecture-style video had the highest number of views out of all categories, with 114,884 views. The median likes/dislikes ratio for the lecture style of videos was 6.5 (IQR 1,12) as compared to technicalbased videos, which had a median likes/dislikes ratio of 1 (IQR 0,4). Although technical videos were the most common style of video $(n=36)$, they had fewer likes $(1$ [IQR $0,4]$ ) and views (253 [IQR 65, 824]).

When comparing video style with dichotomized (good/ excellent versus low quality) GQS (Fig. 3), 64\% of lecturestyle videos versus none of the videos in the 3 other video style categories (technical, testimonial, and other) were deemed good or excellent by neurosurgeon A $(\mathrm{p}<0.001)$. This same trend was seen by all raters; $29 \%-86 \%$ of the lecture-style videos versus $0 \%-6 \%$ of the other video styles were scored as good/excellent quality.

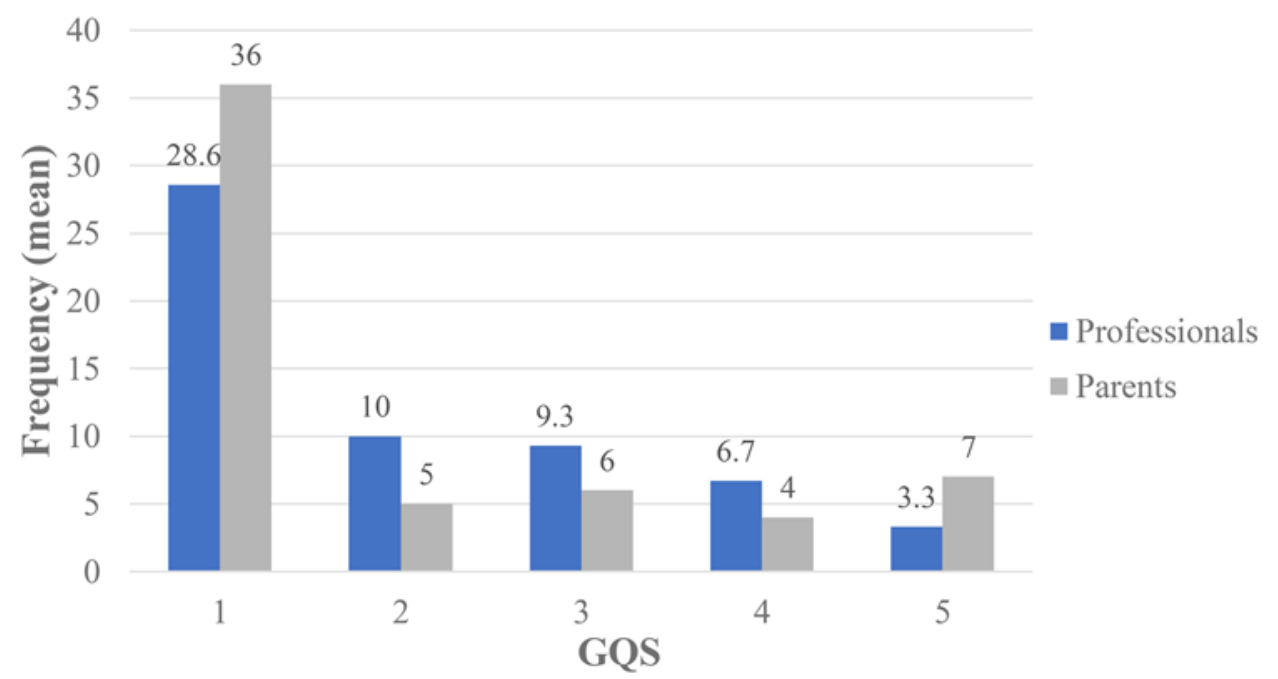

FIG. 2. The comparison between professionals and parents in mean frequency of GQS values. Figure is available in color online only. 
TABLE 3. Metrics of popularity based on video style

\begin{tabular}{lccccccc}
\hline \multicolumn{1}{c}{ Categories } & Likes & Dislikes & Comments & Views & Likes/Views & Comments/Views & Likes/Dislikes \\
\hline Technical, $n=36$ & $1(0,4)$ & $0(0,0)$ & $0(0,0)$ & $253(65,824)$ & $0.002(0,0.01)$ & $0(0,0)$ & $1(0,4)$ \\
\hline Lecture, $n=14$ & $7.5(1,12)$ & $0(0,0)$ & $0(0,1)$ & $896(418,6061)$ & $0.005(0.002,0.01)$ & $0(0,0.0009)$ & $6.5(1,12)$ \\
\hline Testimonial, $\mathrm{n}=2$ & $27.5(25,30)$ & $0.5(0,1)$ & $2.5(0,5)$ & $1612(1444,1781)$ & $0.02(0.02,0.02)$ & $0.001(0,0.003)$ & $27.5(25,30)$ \\
\hline Other, $\mathrm{n}=6$ & $1.5(0,2)$ & $0(0,0)$ & $0(0,0)$ & $418(95,599)$ & $0.002(0,0.004)$ & $0(0,0)$ & $1.5(0,2)$ \\
\hline$p$ value & 0.02 & 0.74 & 0.29 & 0.03 & 0.17 & 0.24 & $\mathbf{0 . 0 1 5}$ \\
\hline
\end{tabular}

Values are expressed as the median (IQR). Boldface type indicates statistical significance.

\section{Discussion}

YouTube is a revolutionary way of sharing information and is beginning to play a key role in knowledge dissemination between healthcare practitioners and patients. Our study evaluated the quality of information resources available on YouTube regarding ETV + CPC for the treatment of pediatric hydrocephalus. In terms of video quality, we found substantial agreement on scoring measures between practicing neurosurgeons and near perfect agreement between the neurosurgeons and a neurosurgical resident. However, there was a disconnect between healthcare providers and the parents of patients with pediatric hydrocephalus in terms of video quality rating. Bridging this gap may improve the delivery of online healthcare resources to patients and their families. Also, the results of this study demonstrate a clear preference for lecture-style videos based on most online popularity metrics and objective rating scales.

\section{Health Communication and Knowledge Dissemination Using YouTube}

Over the past decade, there has been a dramatic rise in the use of social media among healthcare practitioners, patients, and their families. ${ }^{10,29}$ Previous work has found that the primary reason patients or their families seek healthcare information on social media is to increase their knowledge; however, a significant number of patients from the same study cited unreliability of information as a main reason why they would not seek healthcare information on the internet. ${ }^{2}$ This highlights the need for more attention to the quality of information available on websites such as YouTube. This project sought to do just that, as it relied on experts and parents of children with hydrocephalus to evaluate the quality of YouTube videos related to ETV and ETV + CPC. Importantly, the study used objective measures that have been previously validated to rate the videos..$^{4,5,27,28}$

\section{Agreement Between Healthcare Providers and Patients' Families on Video Quality}

We found substantial to near perfect agreement between the practicing neurosurgeons, and between the neurosurgeons and a neurosurgical resident on measures of video quality. Expert analysis for content quality control has been previously identified as an important means of assessing the reliability of online health information. ${ }^{7,8,11}$ However, expert approval of a video does not guarantee that it will be an effective means of improving patient/caregiver knowledge if the end users (patients and families) do not find it a worthwhile resource. Importantly, there was a significant disconnect between neurosurgeons and patients' families regarding the quality of ETV and ETV + $\mathrm{CPC}$ videos. One explanation for this is that video style and presentation may play an important role in whether patients find the resources helpful. A chief motivation for patients and families to access expert-driven healthcare information online is acquiring more knowledge related to their health concerns. ${ }^{2}$ As such, it follows that they will be drawn to lecture-style videos, as we saw in our results. Nonetheless, technical demonstrations have a role too, because they may be well suited to junior healthcare prac-

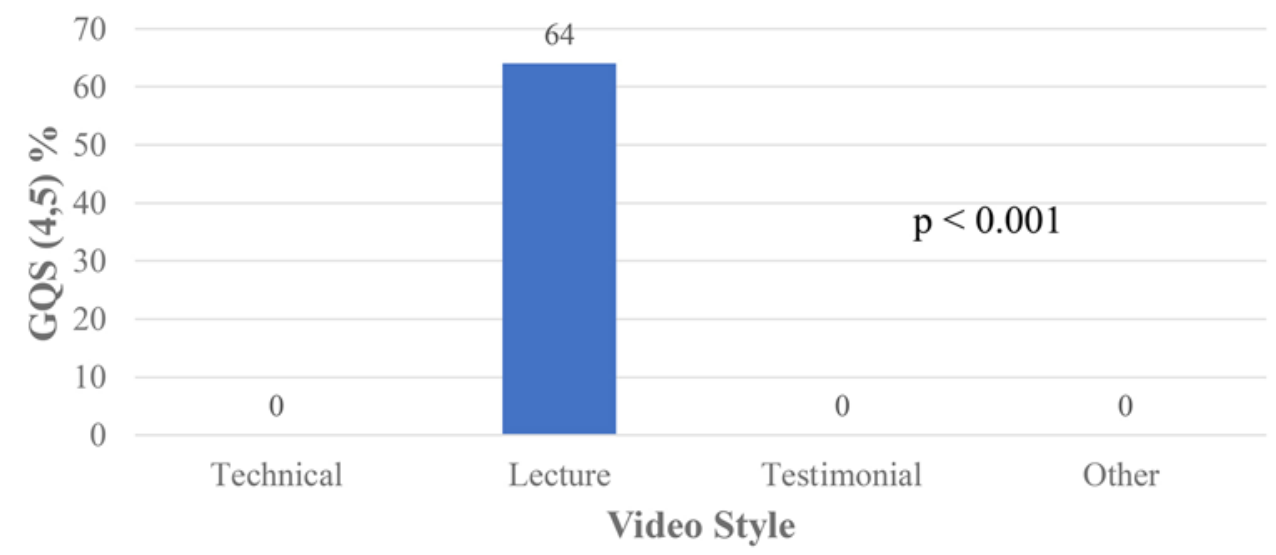

FIG. 3. Percentage of videos with a good or excellent GQS based on video style. Figure is available in color online only. 
titioners trying to learn new procedures. Regardless, no technical videos of ETV and ETV + CPC achieved a good or excellent GQS, indicating that this style of video may not be a valuable information source to either healthcare practitioners or the general public.

We are not stating what videos individuals must access on YouTube. We support having a variety of videos available, including technical videos. However, we are highlighting the fact that our findings suggest that families, trainees, and seasoned neurosurgeons all found lecturestyle videos to be of higher quality. Currently, the bulk of YouTube neurosurgical content is technical videos, which can be interpreted as the favored style of surgeons. Our study does not support that notion, and highlights the fact that videos should have context, descriptions, and a professional walking through the ramifications of the procedure to provide a more global picture.

\section{Popularity Metrics as a Predictor of Video Quality}

Online measures of popularity, such as views or the "like" count, have also been associated with higher healthcare video quality. ${ }^{3,11,26}$ Our study looked beyond likes and views to assess more advanced metrics. The likes/views ratio is meant to evaluate what proportion of watchers have found the video useful. The comments/views ratio assessed how likely video watchers were to interact with the platform by contributing to the comments section. Last, the likes/dislikes ratio evaluated the online community's balance between positive and negative feelings for the video. Again, lecture-based videos were more popular than technical and other styles of videos according to these metrics. When these metrics were analyzed in relation to the GQS, we saw a significant relationship between the likes/dislikes ratio and the GQS. This suggests that the aggregate views of the online community can be used as a rough surrogate for video quality.

\section{Limitations of the Study}

There are a couple of drawbacks to this work that deserve mention. First, only 2 parents of children with hydrocephalus were involved in the rating process; they may not be representative of the larger population. We wanted to include parents who were not associated with either treating institution, so we paired with the Hydrocephalus Association, which found 2 caregivers who were interested in participating in the study. The 2 caregivers did volunteer from an advocacy group, and their children had received treatment for their hydrocephalus. They could have been more familiar with the disease, and it is unknown how much this is generalizable to the parents who are new to the disease and its management. However, their ratings were consistent with YouTube's popularity metrics, which provides some indication that their views may be similar to those of the online community at large. Another limitation to this work was that not all video results from the YouTube search were screened for study inclusion. This was meant to keep the scope of the review within reason and was justified on the basis that previous work identified the fact that online searchers are extremely unlikely to go past the third page of results. ${ }^{14}$ However, it does raise the possibility that some higher-quality videos may have been excluded from the screening process. Finally, it should be noted that the YouTube search was done in June 2017. The internet is a rapidly evolving medium, and new videos could have been added since then that could add to or hinder the quality found.

\section{Conclusions}

In today's society there is an exponential rise in caregivers, professionals, and trainees accessing YouTube videos for educational purposes and communication. However, the content uploaded is unfiltered, with no quality control. We believed it was essential to quantify the quality of videos available and examine the agreement between families, trainees, and professionals. We used expert and patient-family participation to objectively rate the availability of YouTube videos for ETV and ETV + CPC, and we allowed the identification of popularity metrics such as likes, views, and the likes/dislikes ratio as potential markers of video quality. We identified a clear preference for lecture-style videos from both professionals and parents, compared to technical videos. Thus, we are encouraging professionals to use videos with narrative content (lecture style) to achieve a more significant impact on the audience, specifically patients and families.

\section{Acknowledgments}

We thank the two parent scorers for their contribution. As well, we thank Ruksana Rashid for administrative assistance.

\section{References}

1. Anderson M: 5 facts about online video, for YouTube's 10th birthday. Fact Tank. February 12, 2015 (https://www. pewresearch.org/fact-tank/2015/02/12/5-facts-about-onlinevideo-for-youtubes-10th-birthday/) [Accessed January 20, 2020]

2. Antheunis ML, Tates K, Nieboer TE: Patients' and health professionals' use of social media in health care: motives, barriers and expectations. Patient Educ Couns 92:426-431, 2013

3. Backinger CL, Pilsner AM, Augustson EM, Frydl A, Phillips T, Rowden J: YouTube as a source of quitting smoking information. Tob Control 20:119-122, 2011

4. Bernard A, Langille M, Hughes S, Rose C, Leddin D, Veldhuyzen van Zanten S: A systematic review of patient inflammatory bowel disease information resources on the World Wide Web. Am J Gastroenterol 102:2070-2077, 2007

5. Delli K, Livas C, Vissink A, Spijkervet FK: Is YouTube useful as a source of information for Sjögren's syndrome? Oral Dis 22:196-201, 2016

6. Dewan MC, Rattani A, Mekary R, Glancz LJ, Yunusa I, Baticulon RE, et al: Global hydrocephalus epidemiology and incidence: systematic review and meta-analysis. J Neurosurg 130:1065-1079, 2019

7. Eysenbach G, Powell J, Kuss O, Sa ER: Empirical studies assessing the quality of health information for consumers on the World Wide Web: a systematic review. JAMA 287:26912700,2002

8. Fat MJ, Doja A, Barrowman N, Sell E: YouTube videos as a teaching tool and patient resource for infantile spasms. J Child Neurol 26:804-809, 2011

9. Fernell E, Hagberg G, Hagberg B: Infantile hydrocephalus epidemiology: an indicator of enhanced survival. Arch Dis Child Fetal Neonatal Ed 70:F123-F128, 1994 
10. Fox SD, Duggan M: Health Online 2013. Washington, DC: Pew Research Center, 2013 (https://www.pewresearch.org/ internet/2013/01/15/health-online-2013/) [Accessed January 20, 2020]

11. Gabarron E, Fernandez-Luque L, Armayones M, Lau AY: Identifying measures used for assessing quality of YouTube videos with patient health information: a review of current literature. Interact J Med Res 2:e6, 2013

12. Garne E, Loane M, Addor MC, Boyd PA, Barisic I, Dolk H: Congenital hydrocephalus-prevalence, prenatal diagnosis and outcome of pregnancy in four European regions. Eur J Paediatr Neurol 14:150-155, 2010

13. Health on the Net: HONcode Principles. Chêne-Bourg, Switzerland: Health on the Net (https://www.hon.ch/en/) [Accessed January 20, 2020]

14. iProspect: iProspect Search Engine User Behavior Study. Gainesville, FL: University of Florida, 2006 (http://district4. extension.ifas.ufl.edu/Tech/TechPubs/WhitePaper_2006_ SearchEngineUserBehavior.pdf) [Accessed January 20, 2020]

15. Isaacs AM, Riva-Cambrin J, Yavin D, Hockley A, Pringsheim TM, Jette N, et al: Age-specific global epidemiology of hydrocephalus: systematic review, metanalysis and global birth surveillance. PLoS One 13:e0204926, 2018

16. Jeng S, Gupta N, Wrensch M, Zhao S, Wu YW: Prevalence of congenital hydrocephalus in California, 1991-2000. Pediatr Neurol 45:67-71, 2011

17. Kulkarni AV, Drake JM, Mallucci CL, Sgouros S, Roth J, Constantini S: Endoscopic third ventriculostomy in the treatment of childhood hydrocephalus. J Pediatr 155:254-259.e1, 2009

18. Kulkarni AV, Riva-Cambrin J, Browd SR: Use of the ETV Success Score to explain the variation in reported endoscopic third ventriculostomy success rates among published case series of childhood hydrocephalus. J Neurosurg Pediatr 7:143-146, 2011

19. Kulkarni AV, Riva-Cambrin J, Browd SR, Drake JM, Holubkov R, Kestle JR, et al: Endoscopic third ventriculostomy and choroid plexus cauterization in infants with hydrocephalus: a retrospective Hydrocephalus Clinical Research Network study. J Neurosurg Pediatr 14:224-229, 2014

20. Kulkarni AV, Riva-Cambrin J, Rozzelle CJ, Naftel RP, Alvey JS, Reeder RW, et al: Endoscopic third ventriculostomy and choroid plexus cauterization in infant hydrocephalus: a prospective study by the Hydrocephalus Clinical Research Network. J Neurosurg Pediatr 21:214-223, 2018

21. Naftel RP, Safiano NA, Falola MI, Shannon CN, Wellons JC III, Johnston JM Jr: Technology preferences among caregivers of children with hydrocephalus. J Neurosurg Pediatr 11:26-36, 2013

22. Persson EK, Anderson S, Wiklund LM, Uvebrant P: Hydrocephalus in children born in 1999-2002: epidemiology, outcome and ophthalmological findings. Childs Nerv Syst 23:1111-1118, 2007

23. Pew Research Center: Social Media Fact Sheet. Washington, DC: Pew Research Center, 2019 (https://www. pewresearch.org/internet/fact-sheet/social-media/) [Accessed January 20, 2020]
24. Robertson M: 3 metrics ratios to measure YouTube channel success. Tubular Insights. September 18, 2014 (https:// tubularinsights.com/3-metrics-youtube-success/) [Accessed January 20, 2020]

25. Robertson M: Your YouTube likes and dislikes bar: how much does it matter? Tubular Insights. June 5, 2013 (https:// tubularinsights.com/youtube-likes-dislikes-bar-how-muchdoes-it-matter/) [Accessed January 20, 2020]

26. Sood A, Sarangi S, Pandey A, Murugiah K: YouTube as a source of information on kidney stone disease. Urology 77:558-562, 2011

27. Sorensen JA, Pusz MD, Brietzke SE: YouTube as an information source for pediatric adenotonsillectomy and ear tube surgery. Int J Pediatr Otorhinolaryngol 78:65-70, 2014

28. Stellefson M, Chaney B, Ochipa K, Chaney D, Haider Z, Hanik B, et al: YouTube as a source of chronic obstructive pulmonary disease patient education: a social media content analysis. Chron Respir Dis 11:61-71, 2014

29. Van de Belt TH, Berben SA, Samsom M, Engelen LJ, Schoonhoven L: Use of social media by Western European hospitals: longitudinal study. J Med Internet Res 14:e61, 2012

30. Warf BC: Comparison of endoscopic third ventriculostomy alone and combined with choroid plexus cauterization in infants younger than 1 year of age: a prospective study in 550 African children. J Neurosurg 103 (6 Suppl):475-481, 2005

31. YouTube: YouTube for press. YouTube.com (https://www. youtube.com/about/press/) [Accessed January 20, 2020]

\section{Disclosures}

The authors report no conflict of interest concerning the materials or methods used in this study or the findings specified in this paper.

\section{Author Contributions}

Conception and design: Riva-Cambrin, Sader, Kulkarni, Ahmed, Koschnitzky. Acquisition of data: Riva-Cambrin, Sader, Kulkarni, Eagles. Analysis and interpretation of data: Riva-Cambrin,

Sader, Kulkarni, Eagles, Koschnitzky. Drafting the article: RivaCambrin, Sader, Eagles. Critically revising the article: all authors. Reviewed submitted version of manuscript: all authors. Approved the final version of the manuscript on behalf of all authors: RivaCambrin. Statistical analysis: Riva-Cambrin, Sader. Administrative/technical/material support: Riva-Cambrin, Sader, Ahmed, Koschnitzky. Study supervision: Riva-Cambrin.

\section{Correspondence}

Jay Riva-Cambrin: Alberta Children's Hospital, Calgary, AB, Canada. jay.rivacambrin@ucalgary.ca. 